\title{
Erratum: Discriminant analysis for estimation of groundwater age from hydrochemistry and well construction: application to New Zealand aquifers
}

\section{Christopher J. Daughney • Uwe Morgenstern •}

Rob van der Raaij - Robert R. Reeves

Erratum to: Hydrogeology Journal (2009)

DOI 10.1007/s10040-009-0479-2

The rows are mislabelled in Table 3 of the original article.

The corrected version of Table 3 is shown below.

Table 3 Classification functions derived from DA and example calculations. Combined score in bold text corresponds to highest-probability prediction for age category

\begin{tabular}{|c|c|c|c|c|c|c|c|}
\hline \multirow[t]{2}{*}{ Variable } & \multirow[t]{2}{*}{ Units } & \multicolumn{2}{|c|}{ Example input } & \multicolumn{4}{|c|}{ Coefficients for each age category } \\
\hline & & Site 1 & Site 2 & $\leq 10$ & $11-40$ & $41-100$ & $>100$ \\
\hline $\begin{array}{l}\mathrm{Ca} \\
\mathrm{Cl} \\
\mathrm{HCO}_{3} \\
\mathrm{~K} \\
\mathrm{Mg} \\
\mathrm{Na} \\
\mathrm{SO}_{4} \\
\mathrm{Electrical} \text { conductivity } \\
\text { Well depth } \\
\text { Constant } \\
\text { Combined Score }\end{array}$ & $\begin{array}{l}\mathrm{g} \mathrm{m}^{-3} \\
\mathrm{~g} \mathrm{~m}^{-3} \\
\mathrm{~g} \mathrm{~m}^{-3} \\
\mathrm{~g} \mathrm{~m}^{-3} \\
\mathrm{~g} \mathrm{~m}^{-3} \\
\mathrm{~g} \mathrm{~m}^{-3} \\
\mathrm{~g} \mathrm{~m}^{-3} \\
\mathrm{uS} / \mathrm{cm} \\
\mathrm{m} \mathrm{bgs} \\
-\end{array}$ & $\begin{array}{l}75.5 \\
74.6 \\
347.8 \\
1.7 \\
31.3 \\
36.9 \\
0.03 \\
687 \\
150 \\
-\end{array}$ & $\begin{array}{l}19 \\
19 \\
63.5 \\
1 \\
3.8 \\
14 \\
7.7 \\
210 \\
9.1 \\
- \\
\text { Site } 1 \\
\text { Site } 2\end{array}$ & $\begin{array}{l}-39.213 \\
-31.147 \\
-57.123 \\
-6.980 \\
-20.021 \\
-70.347 \\
-4.383 \\
248.281 \\
6.236 \\
-149.124 \\
156.542 \\
\mathbf{1 4 4 . 3 4 7}\end{array}$ & $\begin{array}{l}-40.523 \\
-31.786 \\
-55.442 \\
-7.071 \\
-20.240 \\
-73.988 \\
-4.153 \\
255.339 \\
9.180 \\
-165.608 \\
160.699 \\
143.519\end{array}$ & $\begin{array}{l}-37.816 \\
-30.825 \\
-54.346 \\
-7.580 \\
-17.523 \\
-68.016 \\
-4.416 \\
244.472 \\
9.520 \\
-155.305 \\
164.285 \\
143.765\end{array}$ & $\begin{array}{l}-35.001 \\
-30.209 \\
-48.367 \\
-6.278 \\
-16.701 \\
-63.348 \\
-5.293 \\
232.823 \\
12.996 \\
-156.422 \\
\mathbf{1 6 9 . 5 0 1} \\
139.145\end{array}$ \\
\hline
\end{tabular}

Published online: 19 November 2009

(C) Springer-Verlag 2009

The online version of the original article can be found at http://dx. doi.org/10.1007/s10040-009-0479-2.

C. J. Daughney ( U. Morgenstern • R. van der Raaij •

R. R. Reeves

Institute of Geological and Nuclear Sciences,

P.O. Box 30368, Lower Hutt, New Zealand

e-mail: c.daughney@gns.cri.nz

Tel.: +64-4-5704751

Fax: +64-4-5704600 